\title{
PROJETO DE UM TÚNEL DE VENTO DE BAIXAS VELOCIDADES PARA O ENSINO DE ENGENHARIA DO \\ VENTO
}

\author{
DESIGN OF A LOW-SPEED WIND TUNNEL FOR WIND ENGINEERING TEACHING
}

Gustavo Javier Zani Núñez ${ }^{1}$

DOI: 10.37702/REE2236-0158.v40p394-406.2021

\begin{abstract}
RESUMO
Um Túnel de Vento (TV) de baixas velocidades e de circuito aberto foi projetado, construído e calibrado no Departamento de Design e Expressão Gráfica da Universidade Federal do Rio Grande do Sul. O projeto foi concebido visando a construção de um equipamento de alta qualidade e de baixo custo para o ensino de Engenharia do Vento e para o desenvolvimento de pesquisas acadêmicas. O presente artigo apresenta os fundamentos de projeto e cálculo do Túnel de Vento. O equipamento projetado tem seção de testes quadrada de $0,3 \mathrm{~m}$ x $0,3 \mathrm{~m}$ e comprimento de $0,5 \mathrm{~m}$. A velocidade máxima do vento na seção de testes, sem modelos, é em torno de $19 \mathrm{~m} / \mathrm{s}$. A razão de contração é de 3,36. Duas telas foram utilizadas para minimizar as perturbações do escoamento na seção de testes. A filosofia do projeto é discutida e os métodos de cálculo são descritos, incluindo a estimativa das perdas de carga.
\end{abstract}

Palavras-chave: Túnel de Vento; projeto; ensino de engenharia do vento.

\begin{abstract}
A low-speed open circuit wind tunnel has been designed, constructed, and calibrated at the Department of Design and Graphics at Federal University of Rio Grande do Sul. The design is conceived to be a high quality, low-cost tool for Wind Engineering teaching and academic research. The present paper describes the design calculations of the wind tunnel. The proposed wind tunnel has a test section with cross sectional area of $0.3 \mathrm{~m} \times 0.3 \mathrm{~m}$ and length of $0.5 \mathrm{~m}$. The maximum wind speed is about $19 \mathrm{~m} / \mathrm{s}$ with empty test section. The contraction ratio is 3.36 . Two screens are used to minimize flow disturbances in the test section. The design philosophy is discussed and methods for wind tunnel calculation are outlined, including the pressure losses estimative.
\end{abstract}

Keywords: wind tunnel design, wind engineering teaching.

\section{INTRODUÇÃO}

Neste artigo serão abordados os principais aspectos associados ao projeto do Túnel de Vento do Laboratório de Estudos Aerodinâmicos (LEA), do Departamento de Design e Expressão Gráfica da Universidade
Federal do Rio Grande do Sul. Este equipamento permitirá a realização de medidas em modelos reduzidos diversos. Segundo Stathopoulos e Hajra (2013), a Engenharia do Vento compreende aspectos estruturais e ambientais, estudados em campo, por meio de modelagem física - via Túneis de Vento ou de

\footnotetext{
${ }^{1}$ Professor Associado, Doutor em Engenharia, Universidade Federal do Rio Grande do Sul; gustavo.javier@ufrgs.br
} 
forma computacional - via CFD, ANN e FEM. Dado o caráter multidisciplinar dessa área, a Engenharia do Vento enfrenta vários obstáculos para sua disseminação. Solari (2020) destaca que essa área da Engenharia, considerada relativamente jovem, tem sido difundida principalmente em cursos, programas e escolas independentes. Diante desse cenário, os estudos experimentais em Túnel de vento constituem uma relevante ferramenta para suprir tais obstáculos e assim disseminar o conhecimento para Engenheiros, Arquitetos e Designers.

Os Túneis de Vento (TV) são ferramentas utilizadas para tratar de projetos que envolvam o escoamento de ar em torno de objetos (corpos) e as forças originadas desta interação ar-objeto (NÚÑEZ; LOREDO-SOUZA; ROCHA, 2012). Com o emprego de túneis de vento, é possível medir velocidades globais e localizadas, bem como o campo de pressões na superfície dos objetos.

As partes constituintes de um Túnel de Vento são especificamente projetadas de tal forma que, na seção de testes, o escoamento tenha velocidades médias invariáveis e baixo índice de turbulência (flutuações da velocidade em torno da média).

Os túneis de vento podem ser classificados em dois tipos principais: com circuito aerodinâmico fechado ou aberto. $\mathrm{O}$ escoamento de ar gerado em túneis de vento de circuito aberto tem início no bocal de entrada, acelerando pelo convergente até a seção de testes e sendo conduzido pelo divergente até o ventilador. $\mathrm{O}$ ar utilizado no circuito é o mesmo encontrado no recinto em que se encontra o equipamento. Maiores detalhes sobre vantagens e desvantagens entre os tipos de circuitos aerodinâmicos podem ser encontrados em Barlow, Rae e Pope (1999).

Entre a grande variedade de TV existentes, os de baixa velocidade podem ser considerados aqueles que possuem uso mais genérico. Os equipamentos com seções transversais reduzidas são frequentemente utilizados para pesquisas mais específicas e para fins acadêmicos (BARLOW; RAE; POPE, 1999).

As dimensões globais dos TV dependem, principalmente, do tipo de testes a serem realizados, da velocidade máxima na seção de testes, dos custos de construção, de operação e características dos modelos. Diversos TV utilizados em pesquisas, calibrações e demonstrações possuem área de seção transversal com valores em torno de $0,3 \mathrm{~m}^{2}$ e até mesmo inferiores (BARLOW; RAE; POPE, 1999).

Uma vez estabelecidas as dimensões da seção de testes e a respectiva velocidade máxima, as outras partes podem ser dimensionadas. O TV do LEA foi projetado para reproduzir um escoamento uniforme e suave, ou seja, com distribuição uniforme da velocidade média ao longo da seção transversal e com baixa turbulência. Nestas condições de escoamento, diversos estudos com modelos reduzidos, visualizações do escoamento, medidas de pressões e forças podem ser realizados (BLESSMANN, 1982; 1990).

Além do projeto dimensional do equipamento, é fundamental que seja realizada a estimativa da perda de carga do circuito aerodinâmico, o que terá impacto direto na escolha do ventilador do TV.

\section{FUNDAMENTOS DE PROJETO}

O projeto de um TV é um processo no qual os objetivos são preliminarmente elencados e então são estabelecidos os critérios de projeto. Geralmente, está restringido pelos recursos financeiros disponíveis, custos operacionais, de manutenção e pelo tamanho das instalações (CATTAFESTA; BAHR; MATHEW, 2010). Logo, o projeto de um TV deve ser adaptado para que os objetivos específicos da pesquisa e finalidade de ensaio sejam atingidos. Trata-se de um processo iterativo que tem como ponto de partida as restrições de espaço físico e recursos financeiros, além de outras condicionantes muitas vezes conflitantes.

Um bom projeto de TV deve possibilitar a medição precisa da resposta frente ao vento. $\mathrm{O}$ escoamento deve ser uniforme e suave para que a reprodução de certos fenômenos físicos possa ocorrer. Separações do escoamento devem ser evitadas no interior do circuito aerodinâmico para que não sejam introduzidas instabilidades acompanhadas de ruído aero acústico, além de minimizar as perdas de carga (CATTAFESTA; BAHR; MATHEW, 2010). O bloqueio 
provocado pela presença dos modelos reduzidos no interior do $\mathrm{TV}$, assim como vibrações ocasionadas pelo sistema de propulsão, deve ser mínimo.

O equipamento é composto de diversas seções, cada uma delas com um propósito específico. A finalidade dos ensaios a serem realizados tem relação direta com o tamanho da seção de testes e com a velocidade máxima do vento requerida.

No projeto do TV do LEA, os pontos observados foram os seguintes:

a) limitação de espaço físico;

b) recursos financeiros limitados;

c) túnel de vento de circuito aberto;

d) qualidade do escoamento: uniformidade; e) seção de testes quadrada com maior comprimento possível;

f) velocidade máxima do vento: $\sim 22 \mathrm{~m} / \mathrm{s}$;

g) baixo nível de ruído;

h) tensão de alimentação: $220 \mathrm{~V}$.

As principais condicionantes deste projeto foram os recursos financeiros e o espaço físico disponível. Com esse cenário estabelecido, os outros quesitos foram observados dentro das limitações impostas.

$\mathrm{Na}$ Figura 1 é mostrado o circuito aerodinâmico final do TV de circuito aberto do LEA, com as suas partes constituintes e as principais dimensões.
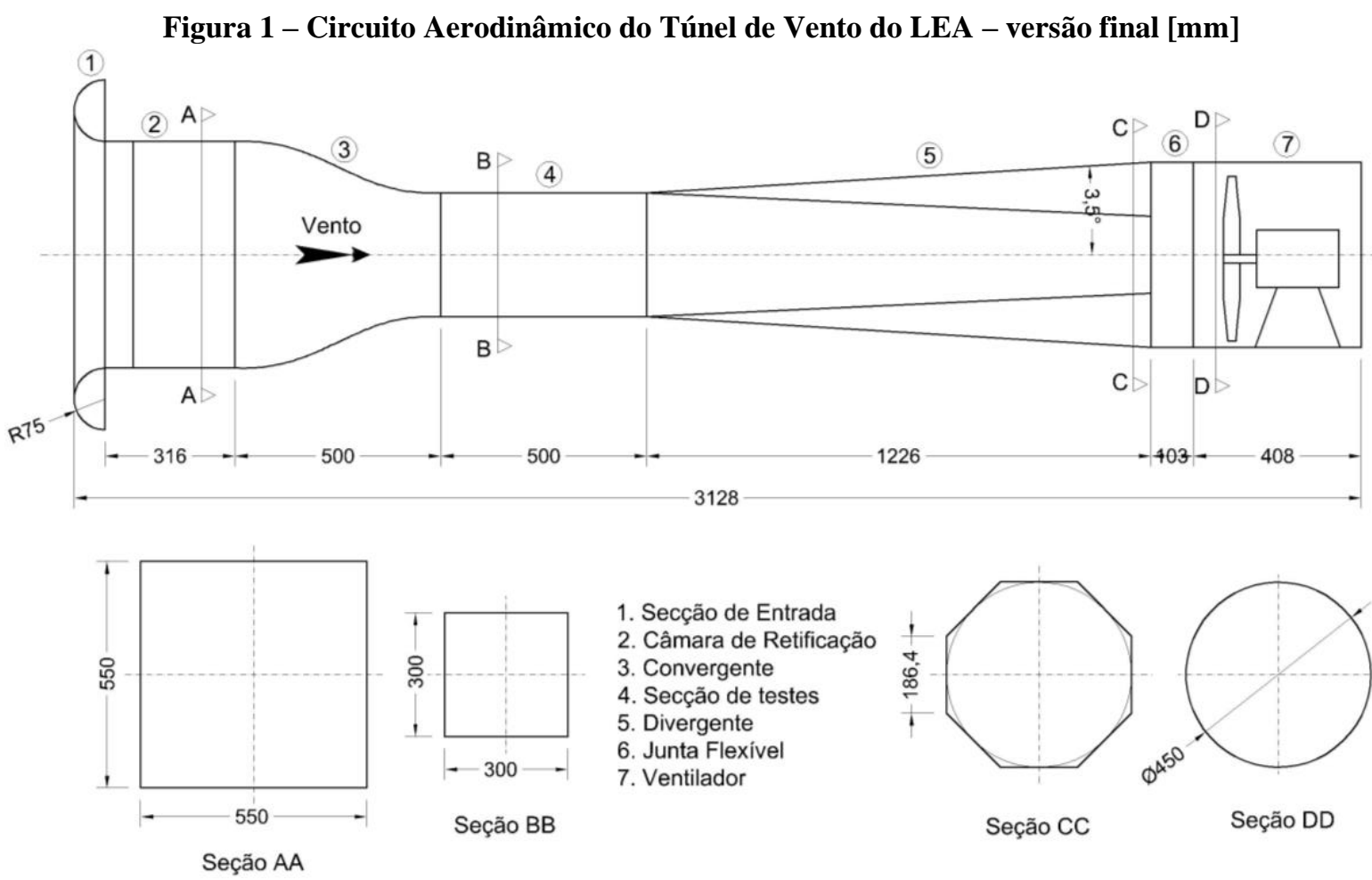

1. Secção de Entrada

2. Câmara de Retificação

3. Convergente

4. Secção de testes

5. Divergente

6. Junta Flexive

7. Ventilador

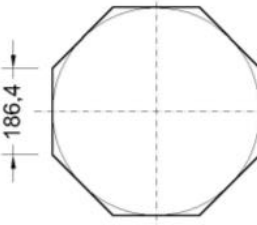

Seção CC

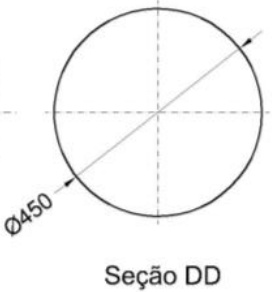

Seção DD

Fonte: elaborada pelo autor.

\section{Processo de projeto}

Este processo interativo tem início na definição das dimensões da seção de testes e a velocidade máxima do vento desejada, possibilitando assim a definição da câmara de retificação. Em geral, são feitas várias modificações e ajustes (entre a razão de contração e respectivo comprimento do convergente) para que sejam obtidas as condições de escoamento estipuladas na seção de testes, desde que os limites das dimensões máximas sejam respeitados. As dimensões da seção de entrada definem o comprimento da câmara de retificação (colmeia e telas). A colmeia é responsável pela retificação do escoamento, gerando uniformidade, enquanto as telas e o próprio comprimento do trecho reduzirão a turbulência na seção de testes. O divergente deve ser projetado para maximizar a 
recuperação da pressão devido às perdas de carga nos trechos a barlavento. Embora um divergente de grande comprimento possibilite uma excelente recuperação da pressão, o limitante passa a ser as dimensões de espaço físico disponíveis. Durante este processo, os limites de parâmetros indicados na literatura técnica devem ser respeitados de tal forma que não ocorram separações do escoamento, evitando-se também um ruído aero acústico. $\mathrm{O}$ sistema propulsor do TV deve fornecer a vazão calculada, além de superar a queda de pressão mantendo-se eficiente. Note-se que as considerações acima dizem respeito a um TV de circuito aberto, no qual o escoamento é captado e expelido no próprio ambiente.

\section{Projeto Geométrico dos Componentes}

Esta seção descreve as orientações de projeto de vários componentes de um TV obtidas a partir de uma revisão bibliográfica em literatura especializada.

$\mathrm{O}$ dimensionamento de um TV inicia pela velocidade do vento desejada na seção de testes (Figura 1). Sabendo-se que as demais partes são diretamente afetadas pelos dados de entrada (dimensões, forma e velocidade máxima da seção de testes) o processo de cálculo pode ser realizado. Para facilitar os cálculos e ajustes necessários durante o dimensionamento do equipamento, foi programada uma planilha eletrônica. Em um único arquivo, cada aba da planilha foi utilizada para o cálculo das dimensões, assim como da perda de carga correspondente de cada uma das partes constituintes do TV. Uma vez estabelecida a perda de carga do conjunto em avaliação, o ventilador pôde ser dimensionado.

\section{Seção de entrada}

O bocal de entrada direciona a entrada de ar, reduzindo a perda de carga e aumentando o volume de ar que entra no TV (HUSSAIN et al., 2011). Bleier (1997) indica que o raio do bocal de entrada $(r)$ deve ser dimensionado de tal forma que $r \geq 0,14 D_{h}$, onde $D_{h}=2 \sqrt{A / \pi}$ é o diâmetro hidráulico da seção de entrada e $A$ é a área da seção transversal da entrada.

\section{Câmara de retificação}

A câmara de retificação tem a finalidade de uniformizar e reduzir a turbulência do escoamento antes do convergente do TV. Hernández et al. (2013) informam que, se é desejável um escoamento de excelente qualidade, a combinação de uma colmeia com telas é fortemente recomendável. Esta configuração requer que a colmeia seja posicionada a barlavento de uma ou de duas telas, todas antes do convergente.

\section{Colmeia}

Segundo Hernández et al. (2013), a colmeia é um dispositivo altamente eficiente na redução das componentes transversais da turbulência do escoamento, o que ocorre quando ele passa por longos e delgados tubos orientados na direção do vento. Entretanto, a colmeia gera turbulência na direção do vento quando apresenta comprimento igual ao seu diâmetro, o que limita o diâmetro das células. Por isso, o comprimento da célula deve ser pelo menos 6 vezes maior do que o correspondente diâmetro (HERNÁNDEZ et al., 2013).

Segundo Brusca, Lanzafame e Messina (2011) os parâmetros chave no dimensionamento de uma colmeia são o comprimento da célula $\left(L_{C O L}\right)$, o diâmetro hidráulico da célula $D_{h-c e l}$ e a porosidade da colmeia $\left(\beta_{C O L}\right)$, a qual é definida pela razão entre a área efetiva de passagem do escoamento e a área total da seção transversal, conforme Equação 1:

$$
\left(\beta_{C O L}=\frac{A_{\text {efetiva escoamento }}}{A_{\text {total secção transversal }}}\right)
$$

Desta forma, de acordo com Mehta e Bradshaw (1979), os dois principais critérios que devem ser verificados são os indicados na Equação 2 (a) e (b):

$$
\text { (a) } 6 \leq\left(L_{C O L} / D_{h-c e l}\right) \leq 8 \text { e (b) } \beta_{C O L} \geq 0,8
$$

A forma da seção transversal das células é usualmente hexagonal (regular), porém, as seções circular e quadrada são também utilizadas por facilidade de construção. 


\section{Telas}

Conforme Brusca, Lanzafame e Messina (2011), sabe-se que o uso de telas reduz principalmente as flutuações de velocidade na direção do vento. Foi demonstrado que uma série de telas com diferentes malhas (grossa, média e fina) é mais eficiente que apenas uma tela fina.

Segundo Mehta e Bradshaw (1979), para que se tenha uma efetiva redução da turbulência longitudinal a tela deve apresentar uma porosidade no intervalo $0,58 \leq \beta_{T} \leq 0,8$. Valores de porosidade $\left(\beta_{T}\right)$ maiores que 0,8 não são eficientes em controlar a turbulência enquanto valores inferiores a 0,58 geram instabilidades no escoamento.

A porosidade é uma função do diâmetro do fio e da densidade da tela. Considerando $\left(d_{f}\right)$ o diâmetro do fio e $\left(l_{m}\right)$ o lado de um quadrado da malha (célula), a densidade da malha $\left(\rho_{\text {malha }}=1 / l_{m}\right)$ relacionada com a porosidade é apresentada na Equação 3.

$$
\beta_{T}=\left(1-d_{f} \rho_{\text {malha }}\right)^{2}
$$

O complemento da porosidade da tela $\left(\sigma_{T}\right)$, é mostrado na Equação 4.

$$
\sigma_{T}=1-\beta_{T}
$$

Mehta e Bradshaw (1979) destacam que as telas utilizadas são normalmente feitas de nylon ou são metálicas, com malhas quadradas ou retangulares.

De acordo com Watmuff (1998 apud CATTAFESTA; BAHR; MATHEW, 2010) as maiores reduções nos níveis de turbulência são obtidas com a colocação da tela de maior porosidade a barlavento e a de menor porosidade a sotavento.

No que diz respeito ao espaçamento entre as telas, Mehta e Bradshaw (1979) sugerem que uma distância entre telas de $0,2 D_{h}$ (diâmetro hidráulico da seção de entrada da câmara de retificação) é suficiente. O mesmo valor de distância sugerido, $0,2 D_{h}$, deve ser observado entre a última tela e o início do convergente. Se o espaçamento for muito pequeno, distorções significativas do escoamento na última tela são esperadas. Por outro lado, se o espaçamento for grande demais, haverá uma indesejável formação de camada limite (MEHTA; BRADSHAW, 1979).

\section{Convergente}

O propósito principal de um convergente é fazer com que determinado volume de ar com baixa velocidade seja acelerado até a entrada da seção de testes. $\mathrm{O}$ escoamento que chega até a entrada da seção de testes deve ser o mais uniforme e suave possível. Porém, o escoamento de ar é inerentemente turbulento devido à presença de acelerações locais e convectivas. Um convergente apropriadamente dimensionado diminuirá as variações tanto da velocidade média quanto da parcela flutuante a baixos níveis (MEHTA e BRADSHAW, 1979).

Brusca, Lanzafame e Messina (2011) destacam que o convergente de um TV é um dos componentes mais difíceis de se dimensionar. A razão de áreas do convergente deve ser a maior possível para que se reduza a perda de carga gerada pela câmara de retificação.

Bell e Mehta (1988) sugerem que os valores típicos de razão de contração (razão entre área da seção transversal de entrada e área da seção transversal de saída do convergente) se situam entre 6 e 10, sendo 8 um valor representativo. Estas considerações são válidas para um TV com área máxima da seção de testes igual a $0,5 \mathrm{~m}^{2}$ e velocidade máxima de $40 \mathrm{~m} / \mathrm{s}$. Barlow et al. (1999) sugerem valores situados entre 7 e 12 com a ressalva de que valores inferiores, ou superiores, aos indicados não são incomuns. Entretanto, segundo Gorecki (1998), TV de baixas velocidades não necessitam de convergentes com grandes valores de razão de contração. Contrações suaves, com razão de áreas entre 2,5 e 6 são usualmente adequadas.

Dimensionar o comprimento do convergente com o maior valor possível é uma alternativa viável para se evitar a separação do escoamento, porém, deve-se cuidar para que a formação da camada limite não seja muito grande (MEHTA; BRADSHAW, 1979).

De acordo com Bell e Mehta (1988) o comprimento total do convergente $(L)$ deve ser igual à altura da seção transversal de entrada do convergente, $L / H_{i}=1$. Em extensa pesquisa 
com polinômios, os autores observaram que a forma mais adequada para o perfil das paredes do convergente foi aquela gerada pelos polinômios de $5^{\mathrm{a}}$ ordem, sendo verificado que, para a razão $L / H_{i}<0,66$, o escoamento se separou próximo à seção de saída, enquanto para a razão $L / H_{i}>1,78$ houve um aumento da espessura da camada limite. Nesta configuração não foram observadas separações do escoamento, indicando que o escoamento gerado tem boa uniformidade. Na Figura 2 são mostradas as curvas polinomiais que representam a forma do convergente.

Figura 2 - Polinômios de ajuste das formas das paredes do convergente

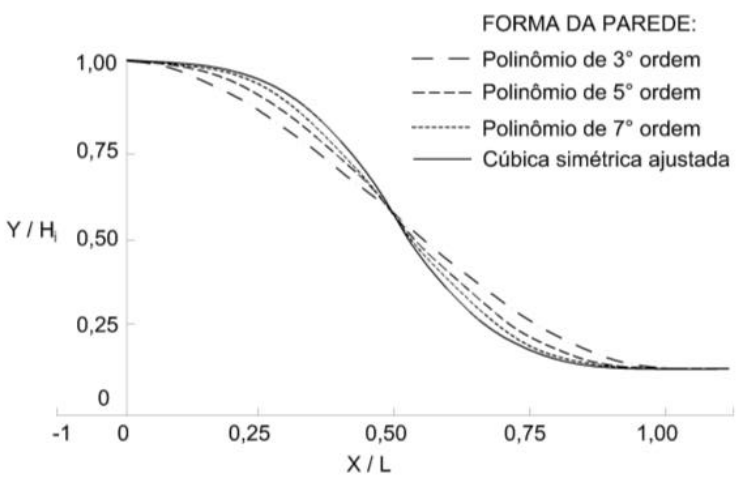

Fonte: adaptada de Bell e Mehta (1988).

A expressão que descreve este polinômio de $5^{\mathrm{a}}$ ordem é mostrada na Equação 5:

$$
y(x)=H_{i}-\left(H_{i}-H_{e}\right)\left[6\left(x^{\prime}\right)^{5}-15\left(x^{\prime}\right)^{4}+10\left(x^{\prime}\right)^{3}\right]
$$

onde:

$H_{i}$ altura da seção de entrada;

$H_{e}$ altura da seção de saída;

$x, y$ coordenadas cartesianas na direção do escoamento e perpendicular à direção do escoamento, respectivamente;

$x^{\prime}$ distância adimensional $X / L$ na direção do escoamento (coincidente com o eixo do TV);

$L$ comprimento do convergente.

A partir da definição das dimensões da seção transversal da seção de testes $(0,3 \mathrm{~m} \times 0,3 \mathrm{~m})$, as dimensões do convergente foram definidas, resultando numa razão de contração de 3,6. As dimensões da seção de entrada do convergente são: $0,55 \mathrm{~m}$ x $0,55 \mathrm{~m}$ e o comprimento do convergente $0,5 \mathrm{~m}$, com respectiva razão $L / H_{i}=0,909$.

A expressão polinomial de $5^{\mathrm{a}}$ ordem que descreve a forma das paredes do convergente do TV do LEA, com perspectiva mostrada na Figura 3, é apresentada na Equação 6:

$$
\begin{aligned}
y= & f(x)=23,88 x^{5}-29,88 x^{4}+9,97 x^{3} \\
& -6 x 10^{-11} x^{2}-5 \times 10^{-11} x-0,275
\end{aligned}
$$

\section{Figura 3 - Perspectiva do convergente do TV}

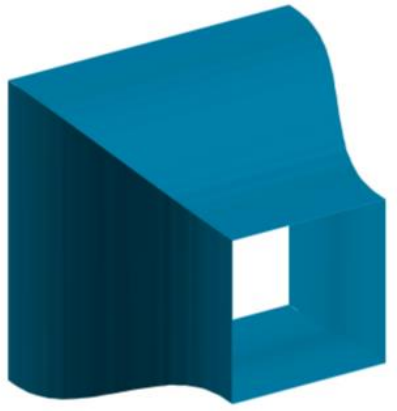

Fonte: elaborada pelo autor.

Com o propósito de calcular o comprimento de material, $L_{\text {linear }}$, necessário para a construção das paredes do convergente, deve ser resolvida a integral definida, apresentada na Equação 7:

$$
L_{\text {linear }}=\int_{0}^{0,5}\left(\sqrt{1+\left[\frac{d y}{d x}\right]^{2}}\right)
$$

Utilizando a função polinomial da Equação 6 na Equação 7 e desprezando os termos de primeira e segunda ordem:

$$
L_{\text {linear }}=\int_{0}^{0,5}\left(\sqrt{1+\left[\left(29,9 x^{2}-119,5 x^{3}+119,4 x^{4}\right)\right]^{2}}\right)(8)
$$

Resolvendo a integral apresentada na Equação 8 o valor de $L_{\text {linear }}=0,52 \mathrm{~m}$.

\section{Seção de testes}

De acordo com Brusca, Lanzafame e Messina (2011), o dimensionamento de um TV deve iniciar pela seção de testes, definindo-se as dimensões e a velocidade máxima do vento. Neste projeto, definiu-se a seção como sendo quadrada de lado $0,3 \mathrm{~m}$ e velocidade máxima do vento de $22 \mathrm{~m} / \mathrm{s}$. Segundo Barlow, Rae e Pope (1999), o comprimento da seção de testes, observado nos projetos de TV, varia entre uma e duas vezes a maior dimensão da seção de testes (altura ou largura). Alternativamente, o comprimento da seção de testes deve estar situado entre 0,5 e 3 vezes o valor do diâmetro hidráulico:

$$
D_{h}=2 \sqrt{A_{S T} / \pi}
$$


onde $A_{S T}$ é a área da seção de testes.

Essa indicação leva em consideração que o escoamento de ar que sai do convergente necessita de um comprimento equivalente a $0,5 D_{h}$ para que esteja uniforme. Além disso, uma seção de testes muito longa, maior que $3 D_{h}$, pode ocasionar um indesejado aumento da camada limite seguido de um descolamento na saída da seção de testes (BARLOW; ERA; POPE, 1999). No projeto do TV do LEA o comprimento da seção de testes foi estabelecido como sendo $1,5 D_{h}$, o que resulta num comprimento de $0,5 \mathrm{~m}$.

Na Figura 4 é mostrada uma perspectiva da seção de testes do túnel de vento do LEA.

Figura 4 - Perspectiva da seção de testes do TV

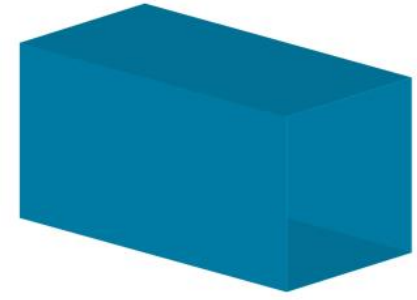

Fonte: elaborada pelo autor.

\section{Divergente}

Segundo Cattafesta, Bahr e Mathew (2010), o propósito do divergente é desacelerar o escoamento oriundo da seção de testes, propiciando a recuperação da pressão estática e reduzindo a carga do ventilador, aumentando assim, a eficiência do TV. A área da seção transversal do divergente deve aumentar gradualmente ao logo do eixo do TV a fim de evitar a separação do escoamento. Conforme Hernández et al. (2013), o divergente tem papel fundamental na qualidade do escoamento na seção de testes. Caso venha a existir uma separação do escoamento, a pulsação de pressão será transmitida a barlavento, avançando na seção de testes, ocasionando perturbações nas pressões e na velocidade do escoamento.

Barlow, Rae e Pope (1999) indicam que, para o dimensionamento de um divergente cônico, o ângulo de expansão cônica equivalente, $\left(\theta_{e}\right)$, mostrado na Figura 5 , pode variar entre 2 e 3,5 (quanto menor, melhor). A razão entre áreas do divergente $A_{R D}$, ou seja, a razão entre a área de saída do divergente
$A_{D \text {-saída }}$, e a área de entrada do divergente, $A_{D-\text { entrada }}$, deve estar situada entre 2 e 3 (quanto menor, melhor). O comprimento do divergente, $L_{D}$, deve ter pelo menos 3 ou 4 vezes o comprimento da seção de testes (BARLOW; RAE; POPE, 1999).

Figura 5 - Parâmetros do divergente

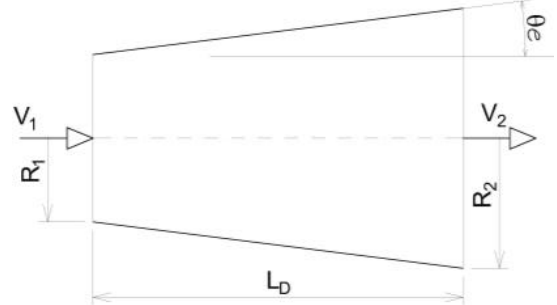

Fonte: adaptada de Barlow, Rae e Pope (1999).

Os parâmetros primários para o dimensionamento de um divergente são o ângulo de expansão cônica equivalente, $\left(\theta_{e}\right)$ e a razão entre áreas. Considere-se um divergente cônico (seção transversal circular) com raio de entrada $R_{1}$, raio de saída $R_{2}$ e comprimento $L_{D}$, identificados na Figura 5.

Considerando-se $\quad R_{1}=D_{h 1} / 2, \quad R_{2}=$ $D_{h 2} / 2$ e o diâmetro hidráulico $D_{h}=2 \sqrt{A / \pi}$, $\left(\theta_{e}\right)$ pode ser definido pela Equação 10:

$$
\theta_{e}=\operatorname{arctg}\left(\frac{R_{2}-R_{1}}{L_{D}}\right)=\operatorname{arctg}\left(\frac{1}{2} \frac{\sqrt{A_{R D}}-1}{L_{D} / D_{h 1}}\right)
$$

Uma vez definido o valor de $\left(\theta_{e}\right)$, e a razão de áreas de entrada e saída do divergente, o seu comprimento pode ser determinado isolando-se $L_{D}$ na Equação 10.

Segundo Mehta e Bradshaw (1979), o escoamento através de um divergente cônico depende de sua geometria, ângulo do divergente, e da forma da seção transversal. O divergente, localizado a sotavento da seção de testes, deve apresentar uma suave expansão. $\mathrm{O}$ ângulo entre as paredes do divergente $\left(2 \theta_{e}\right)$ não deve ser maior do que $5^{\circ}$ para que se obtenha um escoamento mais uniforme e estável. Entretanto, o ângulo ideal para uma melhor recuperação da pressão estática é $10^{\circ}$. A razão entre $A_{D \text {-saída }}$, e $A_{D \text {-entrada }}$ não deve exceder 2,5. É muito importante que se obtenha um escoamento com pouca variação (estável) no divergente, caso contrário, a recuperação da pressão estática tenderá a flutuar gerando flutuações na velocidade do escoamento (MEHTA; BRADSHAW, 1979). 
Considerando-se as indicações propostas por Barlow, Rae e Pope (1999) e Mehta e Bradshaw (1979), o divergente do TV do LEA terá um ângulo de expansão cônica equivalente, $\theta_{e}=3,5^{\circ}$, com razão entre áreas $A_{R D}=1,86 \mathrm{e}$ comprimento $L_{D}=2,45 L_{S T}$. As dimensões principais do divergente são mostradas na Figura 1. Devido a questões de precisão e facilidade de construção do divergente do TV do LEA, optou-se por uma seção octogonal regular na seção de saída, conforme pode ser visto na perspectiva mostrada na Figura 6.

Figura 6 - Perspectiva do convergente do TV

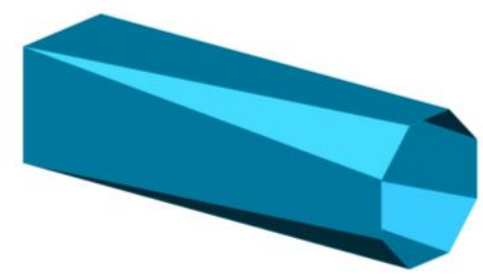

Fonte: elaborada pelo autor.

\section{Perda de Carga dos Componentes}

O sistema de propulsão de um TV deve gerar uma vazão de escoamento capaz de superar as perdas de carga do circuito aerodinâmico. Assim sendo, esta seção descreve a estimativa da perda de carga de cada componente do circuito aerodinâmico para a posterior definição das características do ventilador a ser utilizado.

Em túneis de vento a perda de carga total $\left(\Delta p_{\text {global }}\right)$ é obtida pelo somatório das perdas de carga individuais geradas nas diferentes partes do circuito aerodinâmico. De uma forma geral, em um componente (i) do circuito aerodinâmico a perda de carga correspondente $\left(\Delta p_{i}\right)$ pode ser expressa como sendo o produto entre um coeficiente de perda de carga $\left(k_{i}\right)$, e a pressão dinâmica $\left(P_{D}=1 / 2 \rho V_{i}^{2}\right)$ pode ser estimada geralmente na entrada do componente (i), de acordo com a Equação 11.

$$
k_{i}=\Delta p_{i} /\left(1 / 2 \rho V_{i}^{2}\right)
$$

onde:

$\rho$ massa específica do ar $\left[\mathrm{kg} / \mathrm{m}^{3}\right]$

$V_{i}$ velocidade média do vento $[\mathrm{m} / \mathrm{s}]$

$\left(\Delta p_{i}\right) \quad$ perda de carga (pressão) $[P a]$

Perda de carga na seção de entrada
Tendo a função de reduzir a perda de carga, o bocal de entrada (BLEIER, 1997), apresenta coeficiente de perda de carga conforme Equação 12:

$$
k_{S E}=0,04
$$

\section{Perda de carga na câmara de retificação}

A perda de carga deste trecho engloba o somatório das perdas devidas à colmeia, às telas e ao próprio duto (mesmo processo da seção de testes). Assim, cada elemento apresenta uma sistemática distinta na determinação dos coeficientes de perda de carga.

\section{Colmeia}

De acordo com Eckert, Mort e Jope (1976), a determinação da perda de carga na colmeia considera a razão entre comprimento da célula e seu diâmetro hidráulico e também a porosidade e número de Reynolds $(R e)$ baseado no diâmetro hidráulico da célula. A Equação 13 indica o coeficiente de perda de carga para colmeias com paredes finas:

$$
k_{C O L}=\lambda\left(3+\frac{L_{C O L}}{D_{h-\text { cell }}}\right)\left(\frac{1}{\beta_{C O L}}\right)^{2}+\left(\frac{1}{\beta_{C O L}}-1\right)^{2}
$$

onde $(\lambda)$ pode ser determinado conforme a Equação 14

$$
\lambda=\left\{\begin{array}{lll}
0,375\left(\frac{\Delta}{D_{h-\text { cell }}}\right)^{0,4} R e_{\Delta}^{-1} & \text { para } & R e_{\Delta} \leq 275 \\
0,214\left(\frac{\Delta}{D_{h-\text { cell }}}\right)^{0,4} & \text { para } & R e_{\Delta}>275
\end{array}\right.
$$

O coeficiente de fricção $(\lambda)$ deve ser determinado em função do $(R e)$ baseado na rugosidade superficial $(\Delta)$ do material constituinte.

\section{Telas}

Em túneis de vento, as telas são utilizadas com dois propósitos principais: para a redução dos níveis de turbulência e para a segurança. As telas posicionadas a barlavento e sotavento das hélices do ventilador do TV têm função de segurança. Este tipo de tela impacta consideravelmente na perda de carga por estar posicionada numa região de alta velocidade. Outras telas ficam posicionadas logo após a colmeia, na câmara de retificação, para controle 
de turbulência. Para a estimativa da perda de carga, todas as telas são igualmente consideradas (BARLOW; RAE; POPE, 1999).

De acordo com Eckert, Mort e Jope (1976) dois parâmetros são utilizados para a caracterização das telas, a porosidade $\beta_{T}$, e o número de Reynolds do fio, $R e_{f}=$ $V_{T} d_{\text {fio-T }} / v$. Outro parâmetro, denominado fator de malha $K_{\text {malha }}$, é utilizado para diferenciar as telas. Valores de $K_{\text {malha }}$ indicados por Barlow, Rae e Pope (1999) são, por exemplo, 1,0 para fios metálicos novos, 1,3 para fios metálicos circulares e 2,1 para fios de seda. Na Equação 15 é mostrada a expressão para a estimativa da perda de carga de uma tela.

$$
k_{T}=K_{\text {malha }} K_{R n} \sigma_{T}+\frac{\sigma_{T}^{2}}{\beta_{T}^{2}}
$$

Onde $K_{R n}$ é o fator do $(R e)$ da malha, que pode assumir valores conforme a Equação 16.

$$
K_{R n}=\left\{\begin{array}{cll}
0,785\left(1-\frac{R e_{f}}{354}\right)+1,01 & \text { para } 0 \leq R e_{f}<400 \\
1,0 & \text { para } R e_{f} \geq 400
\end{array}\right.
$$

\section{Perda de carga no convergente}

A perda de carga no convergente pode ser considerada devida apenas à fricção do escoamento com a superfície das paredes (BRUSCA; LANZAFAME; MESSINA, 2011). Levando-se em consideração que as perdas de carga no convergente $\left(k_{C}\right)$, representam algo em torno de $3 \%$ da perda de carga total, os erros decorrentes da estimativa desta perda de carga são menos importantes do que os decorrentes de trechos com maiores velocidades. Segundo Wattendorf (1938 apud

LANZAFAME; MESSINA, 2011) O coeficiente de perda de carga no convergente pode ser estimado de acordo com a Equação 17:

$$
k_{C}=0,32 f_{\text {med. }}\left(\frac{L_{C}}{D_{h-C-e n t r a d a}}\right)
$$

Para dutos com paredes lisas a elevados $(R e)$, pode ser utilizada a lei universal de Prandtl para fricção e assim determinar o fator de fricção $(f)$. $O(f)$ médio entre a entrada e a saída do convergente $\left(f_{\text {med. }}\right)$ pode ser estimado pela Equação 18.

$$
f_{i+1}=\left[2 \log _{10}\left(\operatorname{Re} \sqrt{f_{i}}\right)-0,8\right]^{2}
$$

A Equação 18 pode ser facilmente resolvida de forma iterativa, arbitrando-se um valor inicial de $(f)$. A convergência se dá, geralmente entre quatro e seis iterações, utilizando-se como valor inicial do fator: 1,0. O $(R e)$ a ser considerado é o valor médio entre o valor de entrada e o de saída do convergente.

\section{Perda de carga na seção de testes}

As perdas de carga $\left(\Delta p_{S T}\right)$ em dutos de seção transversal constante são proporcionais ao comprimento $\left(L_{S T}\right)$, ao diâmetro hidráulico $\left(D_{h-S T}\right)$, à massa específica do fluido $(\rho)$ e ao quadrado da velocidade média do escoamento $\left(V_{S T}\right)$, conforme apresentado na Equação 19, onde $(f)$ é o fator de fricção.

$$
\Delta p_{S T}=f\left(\frac{L_{S T}}{D_{h-S T}}\right) \frac{\rho V_{S T}^{2}}{2}
$$

A relação apresentada para a seção de testes é válida para qualquer outra parte do circuito aerodinâmico que tenha seção transversal constante. $O$ coeficiente de perda de carga para seção de testes $\left(k_{S T}\right)$ pode ser escrito conforme Equação 20.

$$
\mathrm{k}_{S T}=f\left(\frac{L_{S T}}{D_{h-S T}}\right)
$$

$O(f)$ deve ser determinado conforme Equação 18. O $(R e)$ deve ser calculado adotando-se como dimensão característica o diâmetro hidráulico $\left(R e=V_{S T} D_{h-S T} / v\right)$.

\section{Perda de carga no divergente}

Num divergente as perdas de carga são geradas por fricção e por expansão do escoamento (BARLOW; RAE; POPE, 1999). No dimensionamento dos divergentes algumas premissas são adotadas. A primeira delas, é que o coeficiente de perda de carga no divergente $\left(k_{D}\right)$ pode ser decomposto como a soma do coeficiente de perda de carga devido à fricção e do coeficiente de perda de carga por expansão, conforme a Equação 21.

$$
k_{D}=k_{f}+k_{\exp }
$$

Assumindo-se que o fator de fricção e a densidade são constantes e, considerando o escoamento em apenas uma dimensão, chegase a uma relação entre o fator de fricção, o 
ângulo de expansão cônica equivalente e razão de áreas do divergente. Assim, o coeficiente de perda de carga devido à fricção $\left(k_{f}\right)$ pode ser determinado através da Equação 22.

$$
k_{f}=\left(1-\frac{1}{A_{R D}^{2}}\right) \frac{f}{8 \operatorname{sen} \theta_{e}}
$$

O fator de fricção $(f)$, é determinado do mesmo modo considerado para seção com área constante, usando $(R e)$ com dados de entrada do convergente (BARLOW; RAE; POPE, 1999).

$O$ coeficiente de perda de carga por expansão $\left(k_{\text {exp }}\right)$, é de difícil determinação sendo utilizados resultados experimentais na sua estimativa. É obtido através do produto de dois fatores, conforme Equação 23 (BARLOW; RAE; POPE, 1999).

$$
k_{\text {exp }}=k_{e}\left(\theta_{e}\right)\left(\frac{A_{R D}-1}{A_{R D}}\right)^{2}
$$

De acordo com Barlow, Rae e Pope (1999) $\mathrm{o}$ fator $\left(k_{e}\left(\theta_{e}\right)\right)$, depende significativamente da seção transversal do difusor. No trabalho de Eckert, Mort e Jope (1976) são apresentados os valores dos coeficientes de perda de carga por expansão em divergentes para seções circular e quadrada. Os valores dos coeficientes são apresentados em função do ângulo de expansão cônica equivalente, conforme as Equações 24 e 25:

$$
k_{e}\left(\theta_{e}\right) \text { circular }\left\{\begin{array}{c}
\text { para } 0^{\circ}<2 \theta<3^{\circ}: \\
\text { para } 3^{\circ} \leq 2 \theta \leq 10^{\circ}: \\
1,033395 \times 10^{-1}-1,19465 \times 10^{-2}(2 \theta) \\
1,70925 \times 10^{-1}-5,84932 \times 10^{-2}(2 \theta) \\
+8,14936 \times 10^{-3}(2 \theta)^{2}+1,34777 \times 10^{-4}(2 \theta)^{3} \\
-5,67258 \times 10^{-5}(2 \theta)^{4}-4,15879 \times 10^{-7}(2 \theta)^{5} \\
+2,10219 \times 10^{-7}(2 \theta)^{6} \\
\text { para } 2 \theta>10^{\circ}: \\
k_{e}\left(\theta_{e}\right) \text { quadrada } \\
\text { para } 0^{\circ}<2 \theta<3^{\circ}: \\
-9,66135 \times 10^{-2}+2,336135 \times 10^{-2}(2 \theta) \\
+5,50704 \times 10^{-3}(2 \theta)^{2}-4,08644 \times 10^{-4}(2 \theta)^{3} \\
-3,84056 \times 10^{-5}(2 \theta)^{4}+8,74969 \times 10^{-6}(2 \theta)^{5} \\
-3,65217 \times 10^{-7}(2 \theta)^{6} \\
\text { para } 3^{\circ} \leq 2 \theta \leq 10^{\circ}: \\
9,62274 \times 10^{-2}-2,07582 \times 10^{-3}(2 \theta) \\
\text { para } 2 \theta>10^{\circ}:
\end{array}\right.
$$

$\mathrm{Na}$ Figura 7 é mostrado o gráfico da variação de $\left(k_{e}\left(\theta_{e}\right)\right)$ em função de $(2 \theta)$. Avaliando a Figura 7, percebe-se que a variação de $\left(k_{e}\left(\theta_{e}\right)\right)$ para valores de $(2 \theta)$ entre $0^{\circ}$ e $4^{\circ}$ apresenta pouca dependência da forma da seção transversal.

Figura 7 - Variação de $\left(k_{e}\left(\theta_{e}\right)\right)$ em função do

ângulo de expansão cônica equivalente $(2 \theta)$

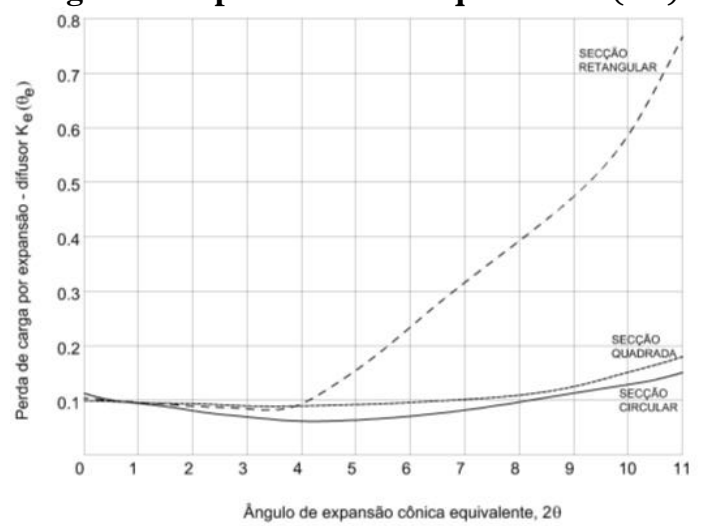

Fonte: adaptada de Eckert, Mort e Jope (1976).

Como neste trabalho existe uma transição da seção quadrada para seção octogonal regular e, não foram encontrados coeficientes de expansão específicos para este caso, será adotado o maior valor obtido entre o calculado para seção quadrada e o calculado para seção circular.

Na Tabela 1 são apresentados, de maneira resumida, os coeficientes de perda de carga parciais estimados por trecho, referentes ao projeto do TV do LEA. Os coeficientes apresentados foram estimados considerando-se $R e=4,4 \times 10^{5}\left(V_{S T}=22 \mathrm{~m} / \mathrm{s}\right)$, Temperatura $=20^{\circ} \mathrm{C}$, Pressão atm $=760 \mathrm{mmHg}$.

Tabela 1 - Coeficientes de perda de carga parciais por trecho, perda de carga e valor \%

\begin{tabular}{lccc}
\hline Trecho & $\boldsymbol{k}_{\boldsymbol{i}}[\mathbf{a d i m}]$ & $\Delta \boldsymbol{p}_{\boldsymbol{i}}[\boldsymbol{P a}]$ & $\boldsymbol{\%}$ \\
\hline Bocal de Entrada & 0,040 & 1,05 & 2,2 \\
\hline $\begin{array}{l}\text { Câmara de } \\
\text { Retificação }\end{array}$ & 0,008 & 0,20 & 0,4 \\
\hline Colmeia & 0,091 & 2,40 & 5,1 \\
\hline Telas (1 $\left.\mathbf{1}^{\mathbf{a}} \mathbf{e} \mathbf{2}^{\mathbf{a}}\right)$ & 1,092 & 28,69 & 61,3 \\
\hline Convergente & 0,007 & 1,05 & 2,2 \\
\hline Seção de Testes & 0,019 & 5,77 & 12,3 \\
\hline Divergente & 0,044 & $\mathbf{7 , 6 4}$ & 16,3 \\
\hline
\end{tabular}

Fonte: elaborada pelo autor. 


\section{Processo de Cálculo da Geometria e da Perda de Carga dos Componentes}

Durante a fase de projeto de um TV é necessário que os cálculos executados durante o dimensionamento sejam feitos de forma confiável e possam ser rapidamente avaliados. Além disso, muitos ajustes são realizados para que as restrições iniciais sejam observadas.

$\mathrm{O}$ projeto da geometria e a estimativa da perda de carga dos componentes do TV do LEA foi assistido por uma planilha eletrônica especificamente elaborada para esta finalidade.

A programação desta planilha exigiu atenção especial às fórmulas inseridas. Foram realizados diversos testes para verificar se a ordem de grandeza dos resultados e os limites de validade das expressões estavam coerentes. Além disso, os resultados calculados foram comparados com valores típicos obtidos na literatura especializada.

Num único arquivo, cada aba foi utilizada para o cálculo das dimensões e da perda de carga correspondente a cada uma das 6 partes constituintes do TV. Essas abas foram dispostas de acordo com a ordem dos componentes do circuito aerodinâmico do TV apresentado na Figura 1. Os dados que permitem o início do processo de projeto devem ser inseridos na aba correspondente à seção de testes. Devem ser informados o valor da velocidade máxima do vento desejada além das dimensões transversais: largura e altura.

A seção de testes apresenta seção transversal quadrada e constante ao longo do seu comprimento, logo, possui a mesma seção transversal de entrada e de saída, em termos de forma e de área. $\mathrm{O}$ trecho adjacente a barlavento é o convergente que, por razões de compatibilidade, terá sua seção transversal de saída quadrada e de mesma área da seção de testes. Ocorrerá o mesmo com trecho adjacente situado a sotavento, o divergente, que terá seção transversal de entrada quadrada e de mesma área da seção de testes.

Como existe um acoplamento entre as dimensões de entrada e de saída entre os trechos adjacentes do circuito aerodinâmico e a vazão do escoamento é constante, a vazão e as dimensões entre as abas adjacentes são iguais.

$\mathrm{Na}$ Figura 8 é apresentada a aba da planilha eletrônica correspondente à seção de testes do $\mathrm{TV}$, na qual é possível identificar os parâmetros utilizados no processo de cálculo da geometria e da estimativa da perda de carga correspondente. Nas células com fundo preto, como por exemplo E2 (largura), E3 (altura) e E8 (velocidade máxima), os valores devem ser fornecidos. Nas células com fundo cinza, como por exemplo E4 (área da seção transversal), E6 (diâmetro hidráulico), E7 (raio hidráulico), N21 (comprimento parcial acumulado) e N22 (perda de carga parcial acumulada), os valores são automaticamente calculados em função dos valores de entrada. Ao posicionar o cursor sobre as células com triângulo vermelho no canto superior direito, como por exemplo C2, C3 e C4, é exibido um comentário para auxiliar na definição do parâmetro associado. Além dos valores numéricos, um gráfico 2D exibe de forma simplificada a geometria em apreciação.

A versão final da planilha de cálculo do TV, composta por 6 abas, pode ser utilizada para novos projetos de TV de baixas velocidades e de circuito aberto diferentes do apresentado neste trabalho. 
Figura 8 - Aba da planilha eletrônica programada para o cálculo da geometria e estimativa da perda de carga referente à seção de testes do Túnel de Vento do LEA

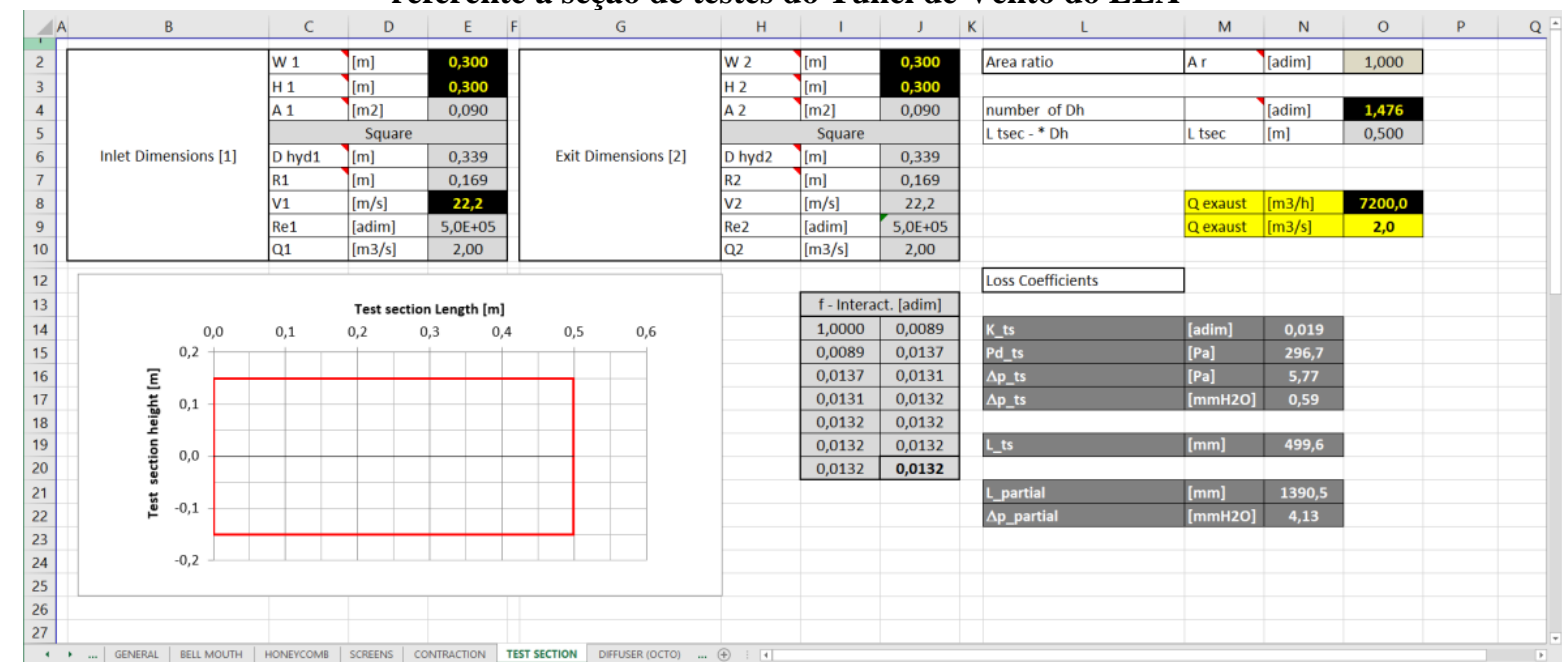

Fonte: elaborado pelo autor.

\section{CONSIDERAÇÕES FINAIS}

Este artigo apresenta os fundamentos de projeto do Túnel de Vento do Laboratório de Estudos Aerodinâmicos (LEA).

Túneis de Vento são ferramentas essenciais para fornecer uma resposta experimental aos problemas que envolvem a interação do vento. Desta forma, o projeto de um TV é um processo iterativo que leva em consideração diversas restrições e orientações.

O projeto do TV foi realizado com base em ampla pesquisa bibliográfica especializada. Os principais parâmetros e orientações de projeto foram selecionados e logo o processo de projeto foi programado em planilha eletrônica. A planilha programada foi repetidamente testada para verificar possíveis erros ou inconsistências. Durante o processo iterativo de projeto foram necessárias diversas alterações de parâmetros e medidas para adaptações às restrições iniciais. $\mathrm{O}$ uso da planilha eletrônica foi extremamente eficaz, fornecendo segurança e agilidade ao processo de cálculo.

O TV servirá como uma ferramenta que possibilitará à comunidade acadêmica, por meio de experimentos interativos dirigidos, uma melhor apropriação de conceitos da mecânica dos fluidos, base da Engenharia do Vento. Por exemplo, ao se visualizar a separação do escoamento em torno de um objeto, com o uso da técnica de injeção de fumaça, podem ser identificadas zonas de separação do escoamento, geralmente associadas a uma grande vorticidade. A partir desta identificação, a geometria ou posicionamento podem ser modificados e uma nova visualização permitirá verificar se a intervenção foi benéfica ou não. Além disso, o TV poderá ser utilizado em pesquisas que visem a avaliação do comportamento aerodinâmico de modelos em escala adequada, tendo em vista que o referido projeto atende aos quesitos técnicos indicados na literatura específica.

\section{AGRADECIMENTOS}

$\mathrm{O}$ autor agradece ao $\mathrm{CNPq}$, ao Prof. Renato Vaz Linn e ao Virtual Design (ViD) da UFRGS pelo apoio.

\section{REFERÊNCIAS}

BARLOW, J. B.; RAE, W. H.; POPE, A. LowSpeed Wind Tunnel Testing. USA: A WileyInterscience Publication, 1999.

BELL, J. H.; MEHTA, R. D. Contraction design for small low-speed wind tunnels. Technical Report 84, Joint Institute for Aernautics and Acoustics, 1988.

BLEIER, F. P. Fan Handbook - Selection, Application and Design. McGrawHill, 1997.

BLESSMANN, J. The Boundary Layer Wind Tunnel of UFRGS. Journal of Wind 
Engineering and Industrial Aerodynamics, Amsterdam, v. 10, p. 231-248, 1982.

BLESSMANN, J. Aerodinâmica das construções. Porto Alegre: Editora Sagra, 1990.

BRUSCA, S.; LANZAFAME, R.; MESSINA, M. Low-speed wind tunnel: design and build. Wind Tunnels: Aerodynamics, Models and Experiment. p.189-220, 2011.

CATTAFESTA, L.; BAHR, C.; MATHEW, J. Fundamentals of Wind-Tunnel Design. Encyclopedia of Aerospace Engineering, R. Blockley and W. Shyy (eds). Chichester, UK: John Wiley \& Sons Ltd, , p. 691-700, 2010.

ECKERT, W. T.; MORT, K. W.; JOPE J. Aerodynamic Design Guidelines and Computer Program for Estimation of Subsonic Wind Tunnel Performance. Washington: NASA: National Aeronautics and Space Administration. 1976.

GORECKI, J. P. Wind tunnel design and applications In: Jubileum Conference on Wind Effects on Buildings and Structures, . 1998, Porto Alegre. Proceedings. Porto Alegre: Brasil, May 25-29, p.1-13, 1998. Riera and Davenport Editors.

HERNÁNDEZ, M. A. G. et al. Design Methodology for a Quick and Low-Cost Wind Tunnel, Wind Tunnel Designs and
Their Diverse Engineering Applications. Noor Ahmed, IntechOpen, 2013.

HUSSAIN, I. Y. et al. Design, construction and testing of low-speed wind tunnel with its measurement and inspection devices. Journal of Engineering, 17. 2011.

MEHTA, R. D. The aerodynamic design of blower tunnels with wide angle diffusers. Progr. Aerosp. Sci., 18, p.59-120, 1977.

MEHTA, R. D.; BRADSHAW, P. Technical Notes: Design Rules for Small Low-speed Wind Tunnels. The Aeronautical Journal of the Royal Aeronautical Society, p 443-449, 1979.

NÚÑEZ, G. J. Z.; LOREDO-SOUZA, A. M.; ROCHA, M. M. Uso do túnel de vento como ferramenta de projeto no design aerodinâmico. DESIGN E TECNOLOGIA, v. 2, p. 10, 2012.

SOLARI, G. Education and dissemination in wind science and engineering. Journal of Wind Engineering and Industrial Aerodynamics, Amsterdam, v. 203, 2020.

STATHOPOULOS, T.; HAJRA, B. Wind engineering education: current state and outlook for the future. In: Proc. 6th Int. Symp. on. Wind Effects on Building and Urban Environment, Tokyo, Japan. 2013.

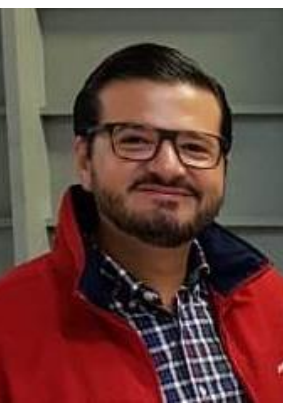

\section{DADOS BIOGRÁFICOS DOS AUTORES}

Gustavo Javier Zani Núñez - Graduação em Engenharia Civil (1998, UFRGS), Mestrado (2001, UFRGS) e Doutorado (2008, UFRGS). Atualmente é Professor Associado I do Departamento de Design e Expressão Gráfica da Universidade Federal do Rio Grande do Sul (UFRGS). Na graduação atua nas disciplinas de Geometria Descritiva para os cursos de Engenharia e Design. Em pesquisa, tem experiência na área de Engenharia Civil, com ênfase em Engenharia do Vento, atuando principalmente nos seguintes temas: Túnel de Vento, Aerodinâmica das Construções - Pontes, Edifícios Altos e Desprendimento de Vórtices. 